\title{
EFECTO DEL ESTRÉS POR SALINIDAD EN CUATRO CEPAS DE Dunaliella salina TEOD. EN EL PERÚ
}

\section{SALT STRESS IN THREE Dunaliella salina TEOD. STRAINS IN PERU}

\author{
Román Felipe Serpa Ibáñez y Abelardo Calderón Rodríguez ${ }^{1}$
}

\begin{abstract}
Resumen
Se estudió el efecto de diferentes niveles de salinidad (1.5 M, 2.5 M, 3.5 M y 4.5 M) en el crecimiento y contenido de pigmentos de cuatro cepas de Dunaliella salina en el Perú.

Los resultados expresaron que hubo una correlación negativa entre: a) La salinidad y la densidad celular a concentraciones mayores de $2.5 \mathrm{M} \mathrm{y} \mathrm{b)} \mathrm{La} \mathrm{salinidad} \mathrm{y} \mathrm{el} \mathrm{contenido} \mathrm{de} \mathrm{pigmentos} \mathrm{(mg/l).}$ La cepa FV-002 presentó el más alto contenido de pigmentos carotenoides y clorofilas.

Palabras clave: salinidad, carotenos, clorofilas, densidad celular, Dunaliella.
\end{abstract}

\section{Astract}

The effect of different levels of salinity $(1.5 \mathrm{M}, 2.5 \mathrm{M}, 3.5 \mathrm{M}$ y $4.5 \mathrm{M})$ were studied on the growth and chlorophyll and carotenoid pigments contents in four Peruvian strains of Dunaliella salina.

The results show a negative correlation between: a) The salinity and the cellular density at concentrations higher than $2.5 \mathrm{M}$ and b) The salinity and the pigments content (mg/l). The strain FV-002 showed the higher pigment content both for carotenoids and for chlorophylls.

Key words: salinity, carotenoids, chlorophylls, cellular density, Dunaliella.

\section{Introducción}

Los estudios fisiológicos de Dunaliella salina se han enfocado principalmente hacia su crecimiento y carotenogenesis, con énfasis en las condiciones que lo optimizan (Ben-Amotz et al., 1982; Borowitzka et al., 1984; Cifuentes et al., 1992). Además de la producción de glicerol, Dunaliella salina produce altas concentraciones de $\beta$-caroteno como respuesta a la limitación de nutrientes (Ben-Amotz et al., 1982; Ben-Amotz \& Avron, 1983; Ben-Amotz \& Morday, 1990), estrés salino (Borowitzka et al., 1990) y alta intensidad de luz (Ben-Amotz \& Avron, 1983; BenAmotz \& Avron, 1989; Ben-Amotz \& Morday, 1990). Dunaliella salina en condiciones hipersalinas $(1.5 \mathrm{M}$ a 3 ó $4 \mathrm{M}$ de $\mathrm{ClNa}$ ) tiene un declive en el crecimiento celular; sin embargo, el flujo de carbón es canalizado hacia el incremento intracelular de glicerol y a un concomitante aumento en la síntesis de isoprenoides plastídicos (Cowan et al., 1992). Bajo condiciones hipersalinas, el glicerol funciona en el citoplasma como un soluto compatible para mantener la integridad de la membrana y proteínas. Al parecer las respuestas celulares al estrés salino son regulatorias y parecen depender de una diversidad de mecanismos ligados a la modificación en el balance del ácido abcísico. Gomez-Pinchetti et al. (1992) y Borowitzka et al. (1990) sostienen que el aumento de la concentración de $\mathrm{ClNa}$ en el medio incide en el aumento de $\beta$-caroteno.

En el presente estudio se evaluó la influencia de diferentes niveles de salinidad en la producción de $\beta$ caroteno y clorofila en condiciones de laboratorio de cuatro cepas silvestres de Dunaliella salina Teod. en el Perú. Por otro lado, el crecimiento se evaluó en términos de densidad celular.

\section{Materiales y Métodos}

Se usaron cuatro cepas de Dunaliella salina provenientes de las salinas de Los Chimus (FV-001), salinas de Huacho (FV-002), salinas de Chilca (FV003) y salinas de Otuma (FV-004), cultivadas en medio enriquecido (Tabla 1). Se probó cuatro diferentes niveles de salinidad $1.5 \mathrm{M}, 2.5 \mathrm{M}, 3.5 \mathrm{M}$ y 4.5 $\mathrm{M}$ versus cuatro cepas.

La salinidad del medio se obtuvo agregando sal industrial a un volumen de agua de mar hasta la concentración deseada. Se consideró que el agua de mar tiene una concentración de sal de $35 \mathrm{~g} / \mathrm{l}$. Aguilar (1995). Así, para llegar a una solución $1.5 \mathrm{M}$ (equivalente $87.55 \mathrm{~g} / \mathrm{l}$ ) se mezclo 52.75 gramos de sal industrial con agua de mar y se enrasó a un litro.

La solución experimental fue preparada con todos los nutrientes y a la misma concentración del medio enriquecido variando solo la salinidad. Se usó como fuente de nitrógeno al $\mathrm{NO}_{3} \mathrm{NH}_{4}$ a una concentración de $2.5 \mathrm{mM} / 1 ; \mathrm{PO}_{4} \mathrm{HK}_{2}$ a una concentración de 0.2 $\mathrm{mM} / \mathrm{l}$; micronutrientes a la misma concentración del medio Guillard, con una variación en la concentración del $\mathrm{H}_{3} \mathrm{BO}_{3}$

En cada tratamiento se usó $150 \mathrm{ml}$ de la solución experimental y se inoculó con $15 \mathrm{ml}$ del cultivo stock mantenido en el mismo medio en fase estacionaria temprana. Cada tratamiento fue repetido tres veces en diferentes lapsos de tiempo $(1 \mathrm{x}, 2 \mathrm{x}$ y $3 \mathrm{x})$. Los 
tratamientos se expusieron a un fotoperíodo de 12:12 (Luz: Oscuridad) y fueron mantenidos a una densidad de flujo fotónico de $\sim 272 \mu \mathrm{E} / \mathrm{m}^{2}$.s, con temperatura semicontrolada (19.9-30.4 $\left.\mathrm{C}^{0}\right)$ y con agitación cada tres días durante 21 días.

Los cultivos fueron periódicamente muestreados y analizadas para determinar la densidad celular y la concentración de pigmentos. Para determinar la densidad celular cada muestra fue fijada con lugol. Los conteos se hicieron empleando una cámara Neubauer estándar de $0.1 \mathrm{~mm}$ de altura y $1 \mathrm{~mm}^{2}$ de área. Para el análisis estadístico de las curvas de crecimiento se realizó un análisis de regresión del promedio de tres observaciones. Se consideró aquella curva con un $\mathrm{R}^{2}$ más grande. Las diferencias significativas en las tasas de crecimiento se hicieron mediante una prueba de $\mathrm{t}$ de student.

Para llevar a cabo la extracción de las clorofilas se tomaron alícuotas del cultivo y se dispusieron en tubos de centrífuga de plástico. Se centrifugo a 5000 g por una hora. El pellet fue lavado directamente con acetona pura $(\sim 3.2 \mathrm{ml})$ y agitado en vortex posteriormente. Se agregó $0.8 \mathrm{ml}$ de agua destilada con el fin de llegar a la concentración de acetona de 80 $\% \mathrm{v} / \mathrm{v}$.

La concentración de carotenoides totales ( $\beta$ caroteno como componente con la mayor concentración en D. salina) fue cuantificada usando $\mathrm{E}_{1 \%}{ }^{480 \mathrm{~nm}}$ (coeficiente de extinción) de 2273 a $480 \mathrm{~nm}$. La concentración de clorofila en mg/l fue cuantificada como lo describe Arnon (1949). Para determinar las diferencias significativas en las concentraciones de los diferentes pigmentos se hizo una prueba de análisis de variancia con arreglo factorial. Las comparaciones se hicieron con la prueba de Tuckey.

Tabla 1. Medio de Cultivo para Dunaliella salina.

Macronutrientes
\begin{tabular}{|l|l|}
\hline \multicolumn{1}{|c|}{$\mathrm{NaCl}$} & 1.5 a $4.5 \mathrm{M}$ \\
\hline Fuente de N & $5 \mathrm{mM} / 1$ de N \\
\hline $\begin{array}{l}\mathrm{KH}_{2} \mathrm{PO}_{4} \text { ó } \\
\mathrm{NaH}_{2} \mathrm{PO}_{4}\end{array}$ & $0.2 \mathrm{Mm} / 1$ \\
\hline
\end{tabular}

Micronutrientes

\begin{tabular}{|l|c|}
\hline $\mathrm{Na}$ EDTA & $4.36 \mathrm{mg} / \mathrm{l}$ \\
\hline $\mathrm{FeCl}_{3}$ & $3.15 \mathrm{mg} / \mathrm{l}$ \\
\hline $\mathrm{Cl}_{2} \mathrm{Mn} \cdot 2 \mathrm{H}_{2} \mathrm{O}$ & $180 \mu \mathrm{g} / \mathrm{l}$ \\
\hline $\mathrm{SO}_{4} \mathrm{Cu} .5 \mathrm{H}_{2} \mathrm{O}$ & $9.8 \mu \mathrm{g} / 1$ \\
\hline $\mathrm{Cl}_{2} \mathrm{Co} . \mathrm{H} 2 \mathrm{O}$ & $10.5 \mu \mathrm{g} / 1$ \\
\hline $\begin{array}{l}\mathrm{Na}_{2} \mathrm{MoO}_{4} \cdot \mathrm{H}_{2} \\
\mathrm{O}\end{array}$ & $6.3 \mu \mathrm{g} / 1$ \\
\hline $\mathrm{SO}_{4} \mathrm{Zn}_{2} \mathrm{H}_{2} \mathrm{O}$ & $22 \mu \mathrm{g} / 1$ \\
\hline $\mathrm{H}_{3} \mathrm{BO}_{3} *$ & $4.45 \mu \mathrm{g} / 1$ \\
\hline
\end{tabular}

\section{Resultados}

Efecto de diferentes niveles de salinidad sobre la densidad celular

Se observa que hay una relación inversa entre la densidad celular promedio y el nivel de salinidad en las cepas de Huacho y Otuma; sucede lo mismo en la fase exponencial en las cepas de Chilca y Los Chimus, pero en ambos casos la menor densidad final se produjo a $2.5 \mathrm{M}$ (Figura 1a)

En todos los casos la menor densidad final promedio en las cuatro cepas se produjo a la mayor salinidad $(4.5 \mathrm{M})$ y la cepa de Otuma presentó la menor densidad. A la salinidad de $3.5 \mathrm{M}$ se alcanzó el siguiente nivel de densidad celular y en tres cepas se alcanzó densidades celulares finales entre $1.5 \times 10^{5} \mathrm{y}$ $2 \times 10^{5}$ células $/ \mathrm{ml}$. De esas tres cepas, la cepa de Otuma fue la que obtuvo la mínima densidad final promedio (Tabla 1.1).

La máxima densidad celular final promedio se dio en la cepa de Huacho $\left(>7 \times 10^{5}\right.$ células $\left./ \mathrm{ml}\right)$. Le siguieron la cepa de Chilca $\left(\sim 5 \times 10^{5}\right.$ células $\left./ \mathrm{ml}\right)$, la cepa de Los Chimus $\left(\sim 3 \times 10^{5}\right.$ células $\left./ \mathrm{ml}\right)$ y la cepa de Otuma $\left(1 \times 10^{5}-1.5 \times 10^{5}\right.$ células $\left./ \mathrm{ml}\right)$ (Tabla 1.1).

En las cepas de Chilca y Huacho, las máximas tasas de crecimiento o productividades celulares se produjeron a $2.5 \mathrm{M}$ de salinidad (0.43230.507 células/ml.día respectivamente) siendo la cepa de Chilca, la que no tuvo diferencias significativas entre las salinidades de 1.5 a $3.5 \mathrm{M}$ (0.3318-0.3494 células/ml.día) Con respecto a las cepas de Los Chimus y Otuma las máximas tasas de crecimiento se produjeron a $1.5 \mathrm{M}$ de salinidad; no habiendo en la cepa de Los Chimus diferencias significativas entre las salinidades de 2.5 y 3.5 M $\quad(0.2777-0.3037$ células/ml.día) y en la cepa de Otuma entre las salinidades de $\begin{array}{lllll}1.5 & \text { y } & 2.5 & \text { M } & (0.4323-0.4005\end{array}$ células/ml.día) (Tabla 1.1).

Tabla: 1.1. Densidad celular (células/ml) en el dia 21 de cultivo en cuatro cepas de Dunaliella salina creciendo a cuatro salinidades

Los Chimus

Chilca

Huacho

Otuma

\begin{tabular}{|r|r|r|r|}
\hline prm 1.5 & \multicolumn{1}{|c|}{ Prm 2.5 } & \multicolumn{1}{c|}{ prm 3.5 } & prm 4.5 \\
\hline 265000 & 299467 & 177767 & 40000 \\
\hline 351667 & 520000 & 172200 & 51700 \\
\hline 724467 & 599433 & 227800 & 58333 \\
\hline 129433 & 112233 & 61100 & 13900 \\
\hline
\end{tabular}

Efecto de diferentes niveles de salinidad sobre el contenido de carotenoides totales

La mayor concentración de carotenoides totales promedio (mg/l) se produjo en la cepa de Huacho a la salinidad de $1.5 \mathrm{M}(1.96563 \mathrm{mg} / \mathrm{l})$ y las menores concentraciones de carotenoides totales promedio se produjeron en el tratamiento de $4.5 \mathrm{M}$ en las cuatro cepas. A esta última salinidad las cepas de Chilca y Huacho fueron las que obtuvieron la mayor 
concentración final $(\leq 0.75 \mathrm{mg} / \mathrm{l})$, seguida por la cepa de los Chimus $(\sim 0.5 \mathrm{mg} / \mathrm{l})$ y la cepa de Otuma $(<0.25 \mathrm{mg} / \mathrm{l})$, siendo esta última la que tuvo la menor concentración de carotenoides totales a esa salinidad (Figura 1b, Tabla2).

En los demás niveles de salinidad, la evolución en la concentración de carotenoides totales tuvo una alta correlación con la curva de crecimiento celular de cada cepa $(\mathrm{r}=0.7862)$.

En casi todos los tratamientos (1.5 M, 2.5M, 3.5 M y $4.5 \mathrm{M}$ ) en las cepas de Chilca, Huacho y Otuma, las series de observaciones que presentaron menores concentraciones de carotenoides totales se dieron en aquellos cultivos que tuvieron temperaturas superiores a $29{ }^{\circ} \mathrm{C}$. Cabe destacar que las más altas temperaturas se dieron durante los meses de verano. En el caso de la cepa de Los Chimus este comportamiento solo sucedió a 2.5 M y 4.5 M. ( Figura 1b,Tabla 2).

Tabla: 2: Concentracion de carotenoides en $\mathrm{mg} / \mathrm{l}$ en el dia 21 de cultivo en cuatro cepas de Dunaliella salina creciendo a cuatro salinidades

\begin{tabular}{|c|c|c|c|c|}
\hline & prm 1.5 & Prm 2.5 & prm 3.5 & prm 4.5 \\
\hline Los Chimus & 1.3878 & 1.7317 & 1.3552 & 0.5159 \\
\hline Chilca & 1.1975 & 1.7408 & 1.7416 & 0.7526 \\
\hline uacho & 1.9656 & 1.3786 & 1.5889 & 0.8346 \\
\hline Otuma & 0.8841 & 0.9253 & 0.5242 & 0.2402 \\
\hline
\end{tabular}

Las mayores tasas de producción de carotenoides o productividades de carotenoides en la cepa de Chilca, Otuma y Chimus se produjeron a 2.5 M (0.2621-0.2122-0.2189 mg/l.día respectivamente) y en la cepa de Huacho a $1.5 \mathrm{M}(0.2523 \mathrm{mg} / \mathrm{l}$.día).Con respecto a las productividades, en la cepa de Los Chimus la mayor productividad se produjo a $2.5 \mathrm{M}$ sin embargo no hubo diferencias significativas entre las salinidades $1.5 \mathrm{M}, 2.5 \mathrm{M}$ y $3.5 \mathrm{M}(0.1776-0.2189$ $0.2104 \mathrm{mg} / \mathrm{l}$.día respectivamente). El mayor nivel de salinidad en todos los casos presentó las menores pendientes es decir tuvieron las menores productividades de carotenoides (Figura 1b).

Cuando se comparó las productividades de las cepas producidas a cada salinidad no hubo diferencia significativa en el intervalo entre $1.5 \mathrm{M}$ a $3.5 \mathrm{M}$ de salinidad (fue un excepción la comparación de la cepa de Los Chimus y Huacho a $1.5 \mathrm{M}$ ). Sin embargo si hubo diferencias significativas a $4.5 \mathrm{M}$ de salinidad entre la cepa de Otuma y las cepas de Chilca y Huacho.

La menor concentración final promedio de carotenoides totales en $\mathrm{pg} /$ célula se produjo en los tratamientos con la salinidad de $1.5 \mathrm{M}$ y $2.5 \mathrm{M}$. y las mayores concentraciones finales se produjeron a 4.5 M (Tabla 2.1). La salinidad de $4.5 \mathrm{M}$ alcanzó la máxima concentración antes de los 10 días y en todo el cultivo su concentración fue mayor a $10 \mathrm{pg} /$ célula.
Este último tratamiento en el día 21 siempre produjo concentraciones promedio mayores al tratamiento con 3.5 M de salinidad.

Tabla 2.1.: Concentracion de carotenoides en pg/célula en el dia 21 de cultivo en cuatro cepas de Dunaliella salina creciendo a cuatro salinidades

\begin{tabular}{l|r|r|r|r|}
\cline { 2 - 5 } & prm 1.5 & prm 2.5 & prm 3.5 & prm 4.5 \\
\cline { 2 - 5 } Los Chimus & 5.409 & 5.9407 & 7.5733 & 13.2561 \\
\cline { 2 - 5 } Chilca & 3.6954 & 3.5579 & 10.098 & 16.3583 \\
\cline { 2 - 5 } Huacho & 2.8427 & 2.5599 & 6.8806 & 14.7039 \\
\cline { 2 - 5 } Otuma & 8.1075 & 8.3072 & 12.4661 & 17.8998 \\
\cline { 2 - 5 } & \multicolumn{2}{|c}{} & \multicolumn{2}{|c}{}
\end{tabular}

Efecto de diferentes niveles de salinidad sobre el contenido de clorofila en $\mathrm{mg} / \mathrm{l}$

Al igual que en la densidad celular y concentración de carotenoides totales, la menor concentración de clorofila se produjo en el tratamiento con la salinidad de $4.5 \mathrm{M}$ en las cuatro cepas. A esa salinidad las cepas de Chilca y Huacho $(\sim 0.25 \mathrm{mg} / \mathrm{l})$ tuvieron concentraciones finales ligeramente mas altas que la que obtuvo la cepa de Los Chimus, siendo la cepa de Otuma la que obtuvo la menor concentración de clorofila $(<0.125)$ (Tabla 3, Figura 1c).

Tabla: 3: Concentración de clorofila en $\mathrm{mg} / \mathrm{l}$ en el día 21 de cultivo en cuatro cepas de Dunaliella salina creciendo a cuatro salinidades

\begin{tabular}{l|r|r|r|r|} 
& prm 1.5 & prm 2.5 & prm 3.5 & prm 4.5 \\
\cline { 2 - 5 } Los Chimus & 1.4277 & 1.48 & 0.8718 & 0.1636 \\
\cline { 2 - 5 } Chilca & 1.0018 & 1.3332 & 0.8462 & 0.275 \\
\cline { 2 - 5 } Huacho & 2.0413 & 1.7712 & 0.8114 & 0.2586 \\
\cline { 2 - 5 } Otuma & 0.7507 & 0.7743 & 0.1638 & 0.1103 \\
\cline { 2 - 5 }
\end{tabular}

A salinidades superiores a $2.5 \mathrm{M}$ se observó que las concentraciones de clorofila fueron siempre menores a la concentración de carotenoides alcanzados a esa salinidad (Figura 1b y 1c, Tabla 2 y $3)$.

En la cepa de Los Chimus, la evolución en el contenido de clorofila en los cuatro niveles presenta una alta correlación con las curvas de crecimiento respectivas manteniendo el mismo orden de las curvas $(r=0.9209)$.

En la cepa de Huacho, en las tres salinidades hay una fase de adaptación, pero después la evolución en el contenido de clorofila es similar a la realizada por la cinética de crecimiento.

En la cepa de Chilca hubo una notoria fase de adaptación, y después un aumento en la concentración de clorofila en los tres tratamientos $(1.5 \mathrm{M}, 2.5 \mathrm{M}$ y $3.5 \mathrm{M})$ que fue similar al comportamiento de la densidad celular a esas mismas salinidades. 
En la cepa de Otuma la evolución en la concentración de clorofila alcanzada por los tratamientos con las salinidades de $2.5 \mathrm{M}$ y $1.5 \mathrm{M}$ es similar (alta correlación) a la evolución de la concentración de carotenoides totales $(\mathrm{r}=0.8911)$.

En todos los tratamientos realizados en las cepas, las concentraciones de clorofila en pg/célula tuvieron una tendencia decreciente en los tratamientos con las salinidades de $2.5 \mathrm{M}, \quad 3.5 \mathrm{M}$ y $4.5 \mathrm{M}$. Las concentraciones en el tratamiento de $1.5 \mathrm{M}$ tuvieron valores casi constantes, y si hubieron variaciones positivas o negativas, estas fueron muy leves (Tabla 3.1). En todos los casos las concentraciones de clorofila son siempre al final menores a $10 \mathrm{pg} /$ célula (Tabla 3.1). La razón carotenoides totales y clorofila en los tratamientos con $3.5 \mathrm{M}$ y $4.5 \mathrm{M}$ en el día 21 es mayor a 1.5 .

Cuando se comparan las concentraciones de clorofila en pg/célula el día 21 en los medios con 1.5 M, 2.5 M, 3.5 y 4.5 M de salinidad no se encuentran diferencias significativas. Sin embargo, cuando se comparan las concentraciones de clorofila entre las cepas, todas tuvieron diferencias significativas ese día $(\alpha=0.01)$.

Tabla: 3.1: Concentración de clorofila en pg/célula en el día 21 de cultivo en cuatro cepas de Dunaliella salina creciendo a cuatro salinidades.

\begin{tabular}{l|r|r|r|r|} 
& prm 1.5 & prm 2.5 & prm 3.5 & prm 4.5 \\
\cline { 2 - 5 } Los Chimus & 6.73 & 5.157 & 4.983 & 4.75 \\
\cline { 2 - 5 } Chilca & 3.33 & 2.78 & 5.06 & 5.773 \\
\cline { 2 - 5 } Huacho & 2.98 & 3.41 & 3.53 & 4.723 \\
\cline { 2 - 5 } Otuma & 6.643 & 7.457 & 6.03 & 7.115 \\
\cline { 2 - 5 }
\end{tabular}

\section{Discusión}

Efecto de diferentes niveles de salinidad sobre la densidad celular

Se sabe que el factor salinidad, disminuye los procesos metabólicos y la replicación celular y aunque todas las variedades de una especie puedan presentar la misma tendencia de crecimiento, es necesario determinar el grado de esta tendencia por medio de la cinética de crecimiento, tasas de crecimiento u otros índices.

En general existe una correlación inversa entre la salinidad y la densidad celular. En la cepa de Los Chimus y Otuma las máximas tasas de crecimiento se produjeron a $1.5 \mathrm{M}$ de salinidad. Sin embargo en las cepas de Chilca y Huacho, las máximas tasas de crecimiento o productividades celulares fueron a 2.5 $\mathrm{M}$ de salinidad.

Se ha determinado que la cepa de $D$. salina aislada del Mar Muerto tiene mayores tasas de crecimiento y densidades finales cuando se cultiva a bajas salinidades (Ben-Amotz \& Avron, 1989). De las cepas analizadas en el presente experimento, solo la cepa de Los Chimus se asemeja a ese reporte, dado que no hay diferencias significativas en las tasas de crecimiento cercanas. Este comportamiento fue también reportado por Aguilar (1995) en esta misma cepa.

Otras cepas de D. salina como la aislada del Gran Lago Salado de Utah tuvo su crecimiento óptimo a la salinidad de $120 \mathrm{o} / \mathrm{oo}(\sim 2 \mathrm{M})$; cabe aclarar que esta salinidad fue menor que la de su hábitat natural donde constituye la flora dominante, aunque crece en el mayor rango de salinidad de todas las especies del genero $(20$ - 350 o/oo; $0.3 \mathrm{M}$ - saturado) (Barbarena \& Montoya, 1990). Diversas investigaciones de cepas de Dunaliella salina de América del Sur usan en sus cultivos salinidades entre 2 y $3 \mathrm{M}$. En el presente experimento las cepas Chilca, Huacho y Otuma tuvieron crecimientos óptimos (tasa de crecimiento y densidad celular) a la salinidad de $2.5 \mathrm{M}$ en forma similar al reporte de Cifuentes et al. (1992).

Ocurre una disminución en las densidades celulares finales cuando se aumenta la salinidad del medio de cultivo (Aguilar, 1995; Araneda et al., 1992; Barbarena \& Montoya, 1990; Ben-Amotz \& Avron, 1989; Borowitzka \& Borowitzka, 1988; Carbajal, 1991; Cifuentes et al., 1992). Este estrés de salinidad influencia la capacidad de $D$. salina para adquirir las fuentes necesarias para su crecimiento y productividad. Típicamente, la fijación de carbono por fotosíntesis se retarda en células expuestas a una variedad de estreses. A pesar de lo dicho anteriormente, en células de $D$. salina hay una estimulación en la tasa de adquisición de carbón inorgánico y se ha determinado la presencia de una anhidrasa carbónica adaptada a altas salinidades (Fisher et al., 1996).

Una respuesta característica de $D$. salina al estrés por salinidad es el ajuste en la concentración intracelular de glicerol por regulación del flujo de carbono entre la producción de almidón en el cloroplasto y la síntesis de glicerol en el citoplasma. Así, el flujo de carbono es canalizado del almidón hacia el glicerol con un concomitante incremento en la biosíntesis de isoprenoides plastídicos.

Desde el punto de vista energético, sobre la base de un estudio teórico de la cantidad de ATP necesaria para la síntesis de material celular en bacterias autotróficas no halofílicas (aproximadamente 30 mmol de ATP por cada 0.2 gramos de biomasa seca), el costo de producir solutos osmóticos orgánicos en organismos halofílicos es enorme (Oren, 1999).

Dado que la cantidad de energía necesaria para la producción de solutos osmóticos quizás exceda grandemente los requerimientos energéticos para la biosíntesis de proteínas, ácidos nucleicos, paredes celulares y otros compuestos, la energía disponible para el crecimiento se ve disminuida cuando uno incrementa la salinidad del cultivo. 
Efecto de diferentes niveles de salinidad sobre el contenido de carotenoides

Las concentraciones de carotenoides totales en $\mathrm{mg} / \mathrm{l}$ y las salinidades del cultivo tuvieron una correlación negativa a salinidades superiores a $2.5 \mathrm{M}$.

De las cuatro cepas, la cepa de Huacho fue la que obtuvo la mayor concentración de carotenoides, además fue la única que obtuvo esa alta concentración a la mas baja salinidad analizada (1.5 M). Mediante el análisis de varianza de las concentraciones de carotenoides totales a los 21 días, se ha demostrado que a las salinidades 1.5 y $2.5 \mathrm{M}$, las concentraciones de carotenoides fueron óptimas. Este comportamiento se asemeja al presentado por la densidad celular. Dadas esas evidencias, cuando el fin de cultivo es obtener altas densidades celulares y/o altas concentraciones de carotenoides totales, las salinidades del cultivo óptimas serian de $1.5 \mathrm{M}$ ó 2.5 M.

En cuanto a las tasas de producción de carotenoides (mg de carotenoides / litro de cultivo. día) entre las salinidades de 1.5 a $3.5 \mathrm{M}$ y en algunos casos 4.5 M (cepa de Huacho) no hay diferencia significativa.

En respuesta al aumento de la salinidad no solo hay una disminución en la tasa de crecimiento (BenAmotz \& Avron, 1983; Ben-Amotz \& Avron, 1989), o en la tasa de fijación de carbono sino una acumulación de carotenoides, clorofila y un aumento en la actividad metabólica del ácido abcísico. También hay una variación en la concentración de glicerol como un soluto compatible en respuesta a los cambios de salinidad (Brown \& Borowitzka, 1979; Cowan et al., 1992).

Las enzimas en $D$. salina involucradas en el metabolismo del ácido abcísico se localizan en el cloroplasto. Se ha demostrado la interrelación entre la actividad del ciclo de la xantofila y la capacidad de las células de $D$. salina de acumular beta-caroteno y producir ácido abcisico. Así, en células estresadas recientemente por el incremento de la salinidad hay un incremento en la epoxidación de algunos componentes del ciclo de la xantofila que se correlaciona con bajos niveles de beta-caroteno y altos niveles de ácido abcisico. Después de un corto lapso de tiempo cuando hay una disminución de la epoxidación y disminución de la concentración de ácido abcísico, se exalta la acumulación de beta-caroteno (Cifuentes et al., 1995).

En el presente análisis, los cultivos expuestos a temperaturas altas produjeron las menores concentraciones de carotenoides en $\mathrm{mg} / \mathrm{l}$. El incremento de la temperatura puede afectar la absorción de nutrientes como los nitratos, sin embargo la absorción de amonio es ligeramente insensible a las variaciones de la temperatura (Reay et al., 1999). Dado que las temperaturas altas producen cambios cinéticos en la toma de otros nutrientes limitantes, estos cambios en los procesos metabólicos podrían explicar mejor el menor contenido de carotenoides. Se sabe que altas temperaturas producen la liberación de compuestos orgánicos al medio circundante. Así mientras mas se incremente la temperatura del cultivo, mas se incrementa la probabilidad de que las células excreten sustancias orgánicas (Giordano et al., 1994). El aumento de esas sustancias y las temperaturas altas incrementan el número de bacterias. Se sabe que las bacterias inducen la nucleación y precipitación de las sales disueltas pudiendo provocar condiciones de deficiencia nutricional (Ventosa et al., 1998).

La acumulación de beta-caroteno hace más fluidas las membranas plasmáticas en el cloroplasto $\mathrm{y}$ favorece el proceso fotooxidativo. Sin embargo la exposición a temperaturas altas y/o alta intensidad luminosa hiperfluidizan demasiado las membranas tilacoidales lo que provoca finalmente su mayor sensibilidad a daños peroxidativos (Havaux, 1998).

Efecto de diferentes niveles de salinidad sobre el contenido de clorofila

La cantidad de clorofila es un indicador del proceso de fotosíntesis. Así, el hecho de que el contenido de clorofila en $\mathrm{mg} / \mathrm{l}$ disminuya al aumentar la salinidad demuestra que la fotosíntesis es inhibida por incremento de la salinidad del cultivo (Cowan et al., 1992).

De las cepas analizadas, la cepa de Huacho presentó las mayores concentraciones de clorofila en el nivel de salinidad mas bajo $(1.5 \mathrm{M})$ en forma similar a lo ocurrido en los carotenoides totales en $\mathrm{mg} / \mathrm{l}$.

La variación en el pH por el incremento de la salinidad (estrés iónico) altera el flujo linear del fotosistema I hacía el flujo cíclico. Esta alteración en el fotosistema I contribuye a la disminución de la proporción NADPH / NADP y a su vez indica una disminución de las actividades biosintéticas (incluida la síntesis de clorofila). El incremento de la salinidad también influye en la asimilación del $\mathrm{CO}_{2}$ a pesar de la aparición de las anhidrasa carbónicas (Fisher et al., 1996). Así, la acumulación de fotosistemas II inactivos podría ser debido a la fotoinhibición causada por la fotosíntesis en condiciones de limitada fijación de $\mathrm{CO}_{2}$.

No solo el incremento de la salinidad influye en la maquinaria fotosintética, sino la intensidad luminosa puede inactivar o destruir las unidades del fotosistema II. Las cantidades de unidades de fotosistema II activas es un indicador de la actividad celular. Así, una baja concentración del fotosistema II implica una baja actividad metabólica.

\section{Referencias Bibliográficas.}

Aguilar CP. 1995. Crecimiento y ciclo de vida del alga Dunaliella salina Teodoresco (Chlotophyta, Volvocales) de las salinas de los Chimus (Ancash) y las salinas de Chilca (Lima). Tesis para obtener el titulo de Licenciado en Biología. Universidad Ricardo Palma. Lima-Perú. 
Araneda P., Tapia I. \& Gómez-Silva B. 1992. Microalgas del Norte de Chile II. Cultivos en medio de bajo costo de dos cepas de Dunaliella salina (Teodoresco, 1905) nativas del desierto de Atacama. Estud. Oceanol. 11:5359.

Arnon DI. 1949. Copper enzymes in isolated chloroplast polyphenyloxidase in Beta vulgaris. Plant Physiol. 24: 115.

Barbarena C. \& Montoya H. 1990. Crecimiento, ciclo de vida y tolerancia a la salinidad de la microalga Dunaliella viridis Teodoresco. Rev. Fac. de Ocean. Pesq. y Cs. Alimentaría.

2: 34-53.

Ben-Amotz A., Katz A. \& Avron M. 1982. Accumulation of beta-carotene in halotolerant algae: purification and caracterization of oof beta-carotene ric globules from Dunaliella bardawil (Chlorophyceae). J. Phycol. 25: 175-178.

Ben-Amotz A \& Avron M. 1983. On the factors which determine the massive beta-carotene accumulation in the halotolerant algae Dunaliella bardawil. Plant Physiol. 72: 593-597.

Ben-Amotz A \& Avron M. 1989. The biotechnology of mass culturing Dunaliella for products of commercial interest. In "Algal and Cyanobacterial Biotechnology", ed. R.C. Creswell, T. A. Rees \& N. Shah. Longman Scientific and Technical Press, Uk. : 90-114.

Ben-Amotz A. \& Morday A. 1990. The biotechnology of cultivating the halotolerant algae Dunaliella for industrial products. Trend in Biotechnol. 8: 121-126.

Borowitzka L.J., Borowitzka M.A. \& Moulton T.P. 1984. The mass culture of Dunaliella salina for fine chemical: the laboratory to pilot plant. Hidrobiologia. 116/117: 115-121.

Borowitzka M.A. \& Borowitzka L.J. 1988. Dunaliella. In "Microalgal Biotechnology". MA Borowitzka and LJ Borowitzka (eds) Cambridge University Press, Cambridge.: 27-58.

Borowitzka L.J., Borowitzka M.A. \& Kessley D. 1990. Effects of salinity increase on carotenoid accumultion in the green algae Dunaliella salina. J. Applied Phycol. 2: 111-119.

Brown A.D. \& Borowitzka L.J. 1979. Halotolerance of Dunaliella. In "Biochemistry and Physiology of
Protozoa". M. Levandowsky and SH Hunter (eds.), 1, $\left(2^{\text {nd }}\right.$ ed.). Acad. Press. New York. 139-190.

Carbajal M.A. 1991. Estudio del ciclo de vida de una cepa chilena de Dunaliella salina (DUNAL) Teod. Tesis para obtener el grado de Magister en ciencias con mención en botánica, Universidad de Concepción. ConcepciónChile.

Cifuentes A.S., Gonzales M., Conejeros M., Dellarosa V. \& Parra O. 1992. Growth and carotenogenesis in eight strain of Dunaliella salina Teod. from Chile. J. Applied Phycol. 4: 111-118.

Cifuentes A.S., Gonzalez M. \& Parra O. 1995. Método para el cultivo de microalgas de ambientes hipersalinos. En "Manual de Métodos Ficológicos"; K. Alveal, M.E. Ferrario. E:C. Olivera y E. Sar (ed.) Universidad de Concepción. Concepción-Chile.: 251-274.

Cowan A.K., Rose P.D. \& Horne L.G. 1992. Dunaliella salina: a model system for studying the response of plant cell to stress. J. of Experimental Botany. 43: 15351547.

Fisher M., Gokhman I., Pich U. \& Zamir A. 1996. A saltresistant plasma membrane carbonic anhidrase is induced by salt in Dunaliella salina. J. Biol. Chem. 271: 17718-17723.

Giordano M., Davis J. \& Bowea G. 1994. Organic carbon release by Dunaliella salina (Chlrophyta) under different growth copndition of $\mathrm{CO} 2$, nitrogen and salinity. J. Phycol. 30: 249-257.

Gomez-Pinchetti J.L., Ramanoz L.K., Fonter A. \& GarciaReina G. 1992. Photosynthetic Characteristic of Dunaliella salina (Chhlorophyceae, Dunaliellales) in relation to beta carotene content. J. Applied Phycol. 4: 11-15.

Havaux M. 1998. Carotenoids as membramne stabilizers in Chloroplasts. Trends in Plant Science. 3: 147-151.

Oren A. 1999. Bioenergetic aspects of halophilism. Microbiol. Mol. Biol. Rev. 63: 334-348.

Reay D.S., Nedwell D.B., Priddle J. \& Ellis-Evans J.C. 1999. Temperature dependence of inorganic uptake: Reduced affinity for nitrate at suboptimal temperatures in both algae and bacteria. Applied Environm. Microbiol. 65: 2577-2584.

Ventosa A., Nieto J.J., \& Oren A. 1998. Biology of moderately halophilic aerobic bacteria. Microbiol. Mol. Biol. Rev. 62: 504-544. 


\section{Figuras citadas en el texto:}

\section{CEPAS DE LAS SALIDAS DE LOS CHIMUS}
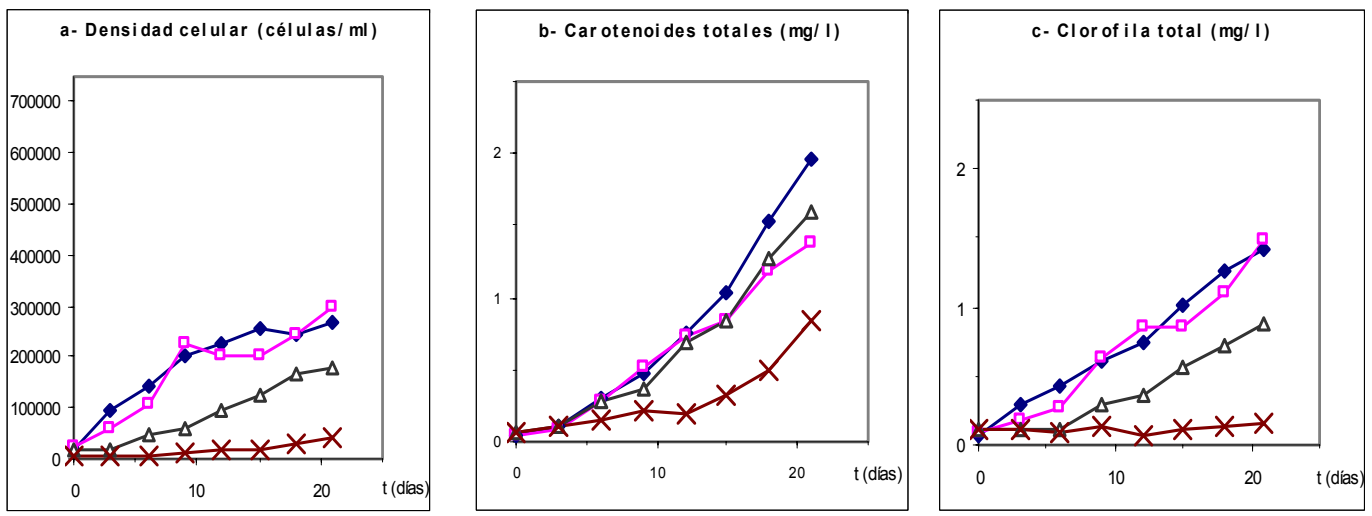

CEPAS DE LAS SALINAS DE HUACHO
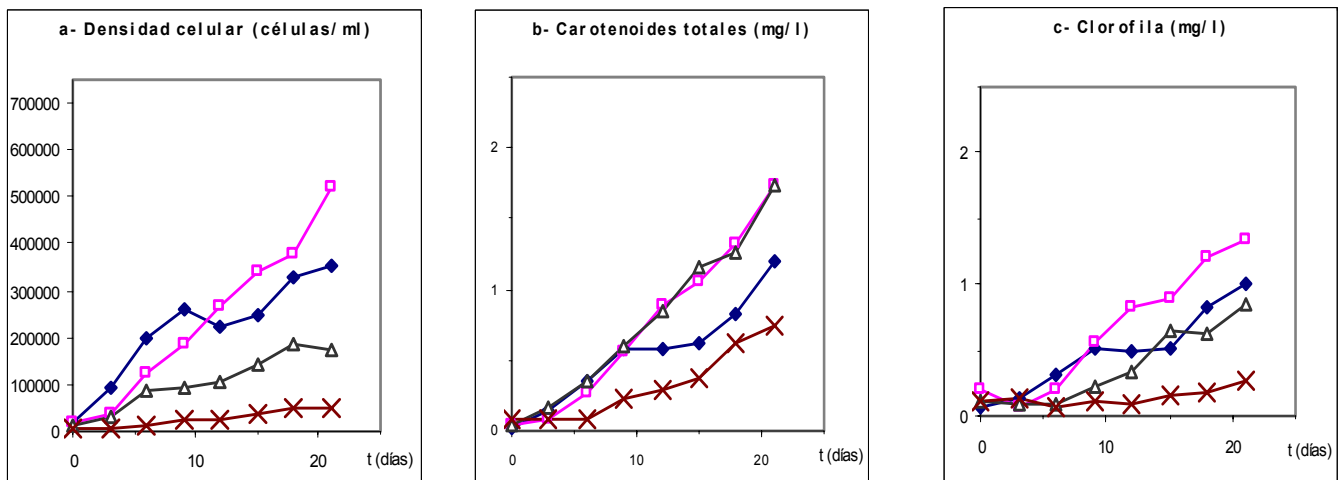

\section{CEPAS DE LAS SALINAS DE OTUMA}
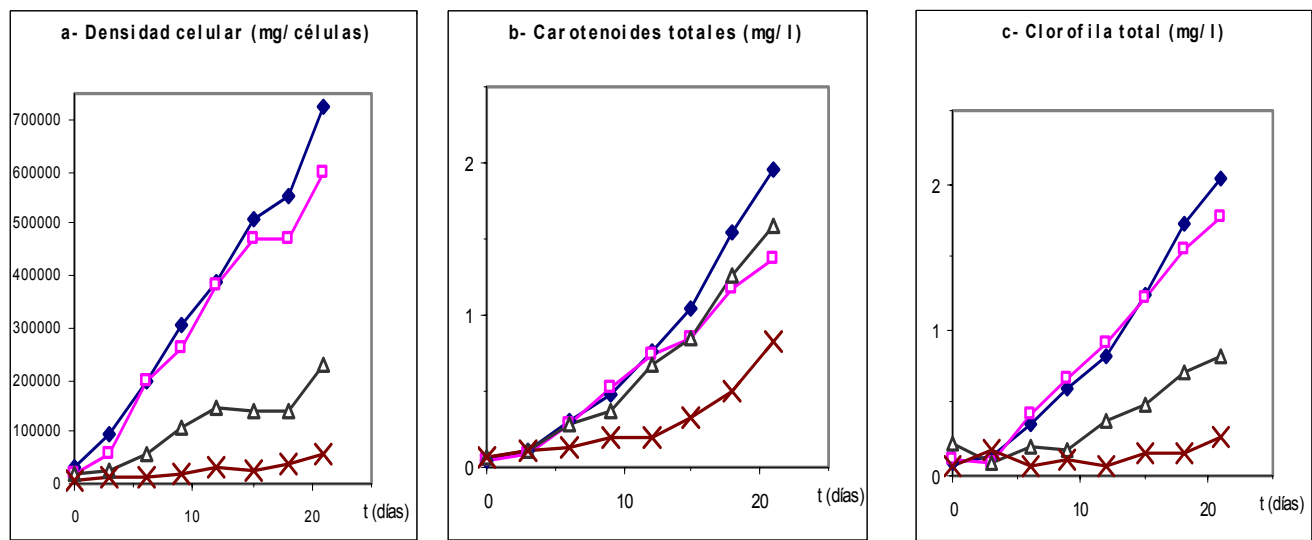

Figura 1. Efectos sobre el crecimiento (a-), contenido de carotenoides en mg/l (b-), contenido de clorofila en mg/l (c-) en cuatro cepas de Dunaliella salina creciendo a cuatro salinidades. Cada curva corresponden al promedio de tres series de observaciones.

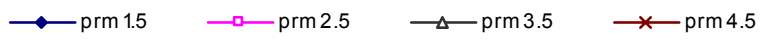

${ }^{1}$ Laboratorio de Fisiología Vegetal de la Universidad Nacional Agraria La Molina, Lima - Perú. Apartado Postal 12-056 Lima 12 Perú. Correo electrónico acalderon@lamolina.edu.pe 